\title{
Psychosocial effects and clinic reflections of the COVID-19 outbreak in patients with acromegaly and Cushing's disease
}

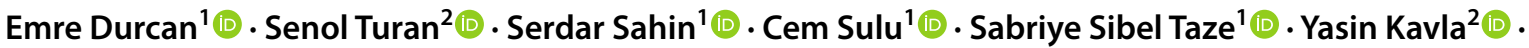 \\ Hande Mefkure Ozkaya ${ }^{1}\left[\right.$ (]) Pinar Kadioglu ${ }^{1}(1)$
}

Accepted: 16 February 2021 / Published online: 5 March 2021

(C) The Author(s), under exclusive licence to Springer Science+Business Media, LLC part of Springer Nature 2021

\begin{abstract}
Purpose Patients with acromegaly and Cushing's disease (CD) may experience significant problems related to the COVID19 outbreak. We aimed to investigate the psychosocial effects of the pandemic and reveal the follow-up characteristics.

Methods The single center, cross-sectional, web-based survey study included patients with acromegaly and CD, PCR-confirmed COVID-19 patients and healthy volunteers without known any chronic disease. The semi-structured sociodemographic data form, The State-Trait Anxiety Inventory (STAI) and Impact of Event Scale-Revised (IES-R) were used.

Results We examined 583 people (217 acromegaly, 127 CD, 102 PCR-confirmed COVID-19 patients and 137 healthy controls). The frequency of abnormal state anxiety and post-traumatic stress disorder (PTSD) were similar in patients with acromegaly and CD and healthy controls, and higher in PCR-confirmed COVID-19 patients than in these three groups ( $p<0.001$ for both). The frequency of abnormal trait anxiety was higher in patients with acromegaly and PCR-confirmed COVID-19 compared to patients with CD and healthy controls ( $p=0.027, p<0.001$, respectively). There were no significant differences between the acromegaly and CD groups in terms of follow-up characteristics and perception of the severity of the COVID-19 outbreak ( $p>0.05$ for all). But, the treatment discontinuation rate was higher in patients with acromegaly than $\mathrm{CD}(\mathrm{p}=0.012)$.

Conclusions Our findings indicate that acromegaly and CD patients are psychologically less affected than PCR-confirmed COVID-19 patients and exhibit similar findings the general population. The clinicians should consider the psychosocial effects, as well as focus on the regular follow-up and medical treatments of these patients during the outbreak.
\end{abstract}

Keywords COVID-19 $\cdot$ Acromegaly $\cdot$ Cushing's disease $\cdot$ Anxiety $\cdot$ Post-traumatic stress disorders

Pinar Kadioglu

kadioglup@yahoo.com

Emre Durcan

dr.durcan@hotmail.com

Senol Turan

senol.turan@istanbul.edu.tr

Serdar Sahin

srdr_shn@hotmail.com

Cem Sulu

drcemsulu@yahoo.com

Sabriye Sibel Taze

sibeltaze@gmail.com
Yasin Kavla

yasinkavla@gmail.com

Hande Mefkure Ozkaya

hndebektas@gmail.com

1 Division of Endocrinology and Metabolism, Department of Internal Medicine, Cerrahpasa Medical School, Istanbul University-Cerrahpasa, Kocamustafapasa Street No: 53, 34098 Fatih, Istanbul, Turkey

2 Department of Psychiatry, Cerrahpasa Medical School, Istanbul University-Cerrahpasa, Istanbul, Turkey 


\section{Introduction}

Coronavirus disease 2019 (COVID-19), which is caused by the severe acute respiratory syndrome coronavirus 2 (SARSCoV-2), has already affected more than 90 million people and caused over 1.9 million deaths worldwide since the first case was confirmed, in Wuhan, China, in the late December of 2019 [1]. SARS-CoV-2 is known to primarily affect the respiratory system, however, studies show that its effects expand also to other organs such as the brain, heart, kidneys, skin, and gastrointestinal system [2-5].

The first confirmed case in Turkey was declared by the government on March 11th, 2020. Unfortunately, 2,336,476 people were diagnosed and 22,981 people died due to COVID-19 in the period between the detection of the first case and January 11th, 2021 [6]. After the confirmation of the first case, many public and private hospitals were selected to be "pandemic hospitals" by the government to combat COVID-19, and new inpatient wards where only COVID-19 patients were treated were established in these hospitals. Additionally, flexible working hours were introduced for healthcare workers, the routine appointments of patients were reduced in all outpatient clinics and nonemergency operations were postponed to prevent the loss of the workforce. Although these new practices initially seemed positive, they have led to uncertainties in the follow-up and treatment processes of individuals with chronic diseases who need regular assessments in hospitals.

Individuals with chronic diseases may experience psychological difficulties during the COVID-19 outbreak for various reasons. In many studies conducted on the general population addressing the psychosocial effects of the COVID-19 outbreak, the presence of chronic diseases has repetitively been identified as risk factors for the development of psychological difficulties [7-9]. Acromegaly and Cushing's disease (CD), like other chronic diseases, cause large numbers of comorbidities, putting individuals at risk for COVID-19. Additionally, the fact that the patients suffering from these diseases have to use medications that necessitates regular follow-up at outpatient clinics. Alongside the difficulties experienced attempting regular follow-ups during the outbreak at outpatient clinics, it can be said that these patients face serious psychosocial hardships.

It is known that psychological difficulties and the psychiatric disorders that develop as a result of these difficulties may negatively affect the course of chronic diseases. In the present study, we aimed to investigate the psychosocial effects of the COVID-19 outbreak on patients with acromegaly and $\mathrm{CD}$ and reveal the follow-up characteristics, risk perception for present diseases and problems with treatment.

\section{Methods}

\section{Participants and procedure}

The single center, cross-sectional, web-based survey study included patients with acromegaly and CD who were followed at the Endocrinology and Metabolism outpatient clinic of Istanbul University-Cerrahpasa, Cerrahpasa Medical School. The positive control group consisted of patients with PCR-confirmed COVID-19 who were followed at inpatient clinic (not in the intensive care unit) of our university hospital between the 1st and 22nd of May 2020 and had in majority mild and moderate disease, and the negative control group consisted of healthy volunteers without any known chronic diseases. The healthy volunteers were recruited through the social media platforms of three different researchers (ED, ST, SST) who were blinded to the results and did not have in common any mutual contacts. Healthcare professionals were excluded from the study.

Healthy volunteers were asked to self-report the presence of any known chronic disorders in the questionnaire and those having any comorbid condition(s) were excluded. Similarly, hospitalized COVID-19 patients who had chronic or comorbid diseases were excluded. Finally, the control groups were formed similar to all of patients in terms of age, marital status and years of education.

An invitation letter, a brief description of the study was sent to participants via social media platforms, such as WhatsApp and Facebook groups between the 27th of April and the 27th of June 2020. Participants gave their consent to participate in the study by clicking the study link sent to them. The waiting period determined to collect the initial responses was one week. A reminder e-mail was sent to participants once a week over the 4-week period. Participants were invited to complete the survey only once and were asked to ignore the reminder e-mails if they had already filled out the questionnaire. All participants who wanted to take the survey were informed that the completion of the questionnaire was anonymous and voluntary.

The study was designed using Google Forms. For this study, a Google account "https://forms.gle/ti6SzwnYGk EsdYrb8 was set up and managed by the lead researcher to collect survey responses. The responses to the questionnaire were collected anonymously and recorded on the designed platform. The questionnaire contained three parts: part I consisted of 30 questions about sociodemographic data form prepared by the authors, part II contained the StateTrait Anxiety Inventory (STAI I-II) and part III contained the Impact of Event Scale-Revised (IES-R). The estimated time to complete the survey was between 13 and $18 \mathrm{~min}$. 


\section{Measures}

\section{Sociodemographic data form}

This data form consisted of questions about the individuals' age, sex, marital status, education status and employment status, number of people living together in the participants' home, their comorbidities, their follow-up characteristics, risk perception for present diseases and problems with treatment in the COVID-19 outbreak. Questions were also posited to gauge their positivity towards COVID-19 and the number of individuals in their family who died due to COVID-19.

\section{State-trait anxiety inventory (STAI S-T)}

The State-Trait Anxiety Inventory (STAI) was used to measure the presence and severity of existing anxiety symptoms and general anxiety tendencies. It included two subscales (state and trait) consisting of a 20 -item self-report questionnaire. First, the state-anxiety subscale (STAI-S) evaluated the current state of anxiety and asks how participants feel "right now". Then, the trait-anxiety subscale (STAI-T) evaluated relatively stable aspects of "anxiety tendencies". Increased scale scores showed the presence of higher levels of anxiety. The scores for the STAI questionnaire ranged from 20 to 80 points and was split into four groups: no anxiety (0-20), mild (21-39), moderate (40-59), and severe anxiety (60-80). An abnormal STAI score was subject when the participants' score was $\geq 40$.

The validity and reliability of the Turkish version of the scale was evaluated by Oner and Le Compte [10]. Cronbach's Alpha internal consistency reliability coefficients were found to be between 0.94 and 0.96 for the state-anxiety, and between 0.83 and 0.87 for the trait-anxiety subscales.

\section{Impact of event scale-revised (IES-R)}

The Impact of Event Scale-Revised (IES-R) was developed by Weiss and Marmar, as a 5-point Likert-type inventory (ranging from 0 to 4 ) that assessed symptoms of posttraumatic stress in relation to any event [11]. IES-R has been repeatedly used in the literature to assess posttraumatic stress disorder after various traumatic situations [12-15]. In the present study, as in various others, it was used to measure stress after the COVID-19 pandemic [16-19]. It consisted of a 22-item self-report questionnaire and three subscales (intrusion, avoidance and hyperarousal). Increased scale scores showed the presence of higher symptoms of posttraumatic stress disorder. Although the original scale did not have a cut-off value, Creamer et al. later set 33 as the IES-R score cut-off value to indicate the presence of PTSD (posttraumatic stress disorders) [20]. In our study, we evaluated our results according to the IES-R total, subscale scores and the IES-R cut-off value (total scores $\geq 33$ ) as indicators of the presence of PTSD.

The validity and reliability of these scales in Turkish was evaluated by Corapcioglu et al. [21]. Cronbach's Alpha internal consistency reliability coefficients were found as 0.94 for the whole group and between 0.87 and 0.94 for the various study groups. We used the validated Turkish version of the questionnaire with one slight modification (we replaced the word "outbreak" for "event").

\section{Ethical issues}

The study was approved by the local ethics committee of the Istanbul University-Cerrahpasa, Cerrahpasa Medical Faculty (Decision No: 56,321 dated 22 April, 2020). And all procedures performed in studies involving human participants were in accordance with the ethical standards of the institutional and/or national research committee and with the 1964 Helsinki declaration and its later amendments or comparable ethical standards.Informed consent was obtained from all individual participants included in the study.

\section{Data analysis}

Statistical analyses were performed using the Statistical Package for the Social Sciences (SPSS) software (version 21.0). Data were first analyzed for normality using the Kolmogorov-Smirnov test. Continuous variables were expressed as mean \pm standard deviation (SD) and/or medians [interquartile range (IQR)]. Student's $t$-test or analysis of variance (ANOVA) were used to compare means between groups with normal data distributions. Medians were compared using the Mann-Whitney U test and the Kruskal-Wallis test. Spearman's rank order test and Pearson's correlation test were used to calculate the correlation coefficients between continuous variables. Frequencies were compared using Pearson's and Fisher's exact tests. The results were evaluated at a $95 \%$ confidence interval, a p-value $<0.05$ was considered statistically significant.

\section{Results}

\section{Response rate}

In total, we sent a study link to 1858 participants (664 acromegaly, $398 \mathrm{CD}, 594$ healthy controls and 202 PCRconfirmed COVID-19 patients). We collected 775 responses in the survey, 217 from acromegaly patients (response rate $32.7 \%$ ), 127 from CD patients (response rate 31.9\%), 303 from healthy controls (response rate 51\%), 128 from 
PCR-confirmed COVID-19 patients (response rate 63.4\%). 137 healthy controls and 102 PCR-confirmed COVID-19 patients were selected in accordance with the match criteria. Finally, data analysis was performed on 583 participants (Fig. 1).

\section{Participants' characteristics}

The sociodemographic characteristics of participants in each group are summarized in Table 1. The comorbid diseases in patients with acromegaly and CD are shown in Table 2 .

\section{Comparison of the groups in terms of STAI and IES-R scores}

The comparison of STAI-S and STAI-T scores between groups were shown in Table 3. While,patients with acromegaly and PCR-confirmed COVID-19 had higher trait anxietyscores than controls, state anxiety scores were highest in the patients with PCR-confirmed COVID-19. Although, patients with $\mathrm{CD}$ and healthy controls had similar frequency of abnormal trait anxiety $(p=0.913)$, this rate was higher in patients with acromegaly and PCR-confirmed COVID19 than controls. Also, we determined that COVID-19-time course caused a low and similar rate of abnormal state anxiety in patients with acromegaly, $\mathrm{CD}$ and in healthy controls $(p=0.974)$, and there was a frequency of heightened state anxiety in PCR-confirmed COVID-19 patients (Fig. 2).

In terms of whether the outbreak was perceived to cause PTSD, the mean IES-R total and subscales scores were higher in PCR-confirmed COVID-19 patients (Table 3). Also, we determined that COVID-19-time course caused a low and similar frequency PTSD in patients with acromegaly, $\mathrm{CD}$ and in healthy controls $(\mathrm{p}=0.605)$ (Fig. 3).

Although the correlation was weak, the presence of state and trait anxiety caused by COVID-19 and the perception of the outbreak as PTSD decreased with increasing age $(\mathrm{p}=0.035, r=0.11 ; \mathrm{p}=0.045, r=0.11 ; \mathrm{p}=0.041, r=0.11$, respectively).

\section{Follow-up characteristics and the risk perception of patients during the COVID-19 outbreak}

Patients' follow-up characteristics, risk perception for present diseases and treatment problems during the COVID-19 outbreak are shown in Table 4. There were no significant differences between the acromegaly and $\mathrm{CD}$ groups in terms of these parameters except the discontinuation rate of medical treatments. This rate was higher in patients with acromegaly than in patients with CD $(19.4 \%$ vs. $10.2 \%, \mathrm{p}=0.012)$.

There were no significant differences between groups for all IES-R and STAI scores, when patients with acromegaly and with $\mathrm{CD}$ were separately divided into two groups according to the presence of comorbidities, perception of the severity of the COVID-19 outbreak, considering acromegaly and $\mathrm{CD}$ and its treatment as a risk factor during the pandemic and thinking that missing a clinic appointment was a problem $(\mathrm{p}>0.05$ for all).

\section{COVID-19 outbreak-related characteristics of the participants}

In both the acromegaly and CD groups, one person had COVID-19 and recovered. Nobody was hospitalized for any reason in these groups. The number of PCR-confirmed COVID-19 individuals in their families were $19(8.8 \%)$ in the acromegaly group, $11(8.7 \%)$ in the CD, $49(48 \%)$ in the PCR-confirmed COVID-19 patient group, and $11(8 \%)$ in the controls. Death from COVID-19 was not present in any of the four groups. The number of healthcare professionals included in the family members of these groups were similar (16.6\% in acromegaly, $15 \%$ in CD, $18.8 \%$ in PCR-confirmed COVID-19 patients and $21.2 \%$ in controls, $\mathrm{p}=0.373$ ).

The number of individuals who thought the outbreak was serious was $160(73.7 \%)$ in the acromegaly, $101(79.5 \%)$ in the CD, 77 (75.5\%) in the PCR-confirmed COVID-19 patient group and 96 (70.1\%) in the control group. There were no significant differences between the groups $(p=0.209)$.

\section{Discussion}

In the present study, we investigated the effects of the outbreak on the psychological conditions of patients suffering from acromegaly and $\mathrm{CD}$, two important chronic endocrinological diseases. We found that PCR-confirmed COVID-19 patients had higher levels of state anxiety and PTSD caused by the outbreak. The state anxiety levels of patients with acromegaly and CD were similar to those of healthy controls. We also observed lower frequency PTSD in the three other groups when compared with the PCRconfirmed COVID-19 patients. Additionally, abnormal trait anxiety was higher in patients with acromegaly and PCRconfirmed COVID-19 in comparison to patients with CD and healthy controls.

Early studies that investigated the psychological effects of COVID-19 had shown that this disease increased anxiety levels in the general population [7, 22-24]. The prevalence of anxiety was between 31.9 and $33 \%$ in different populations studied [25, 26], and $45.1 \%$ in Turkey [8]. In our study, we determined that $58.4 \%$ of healthy controls had abnormal trait anxiety and $49.6 \%$ had abnormal state anxiety, higher than the rates found in the general population. Due to the unique cultural characteristics of the Turkish population, such as close social relations and strong kinship ties, Turkish 


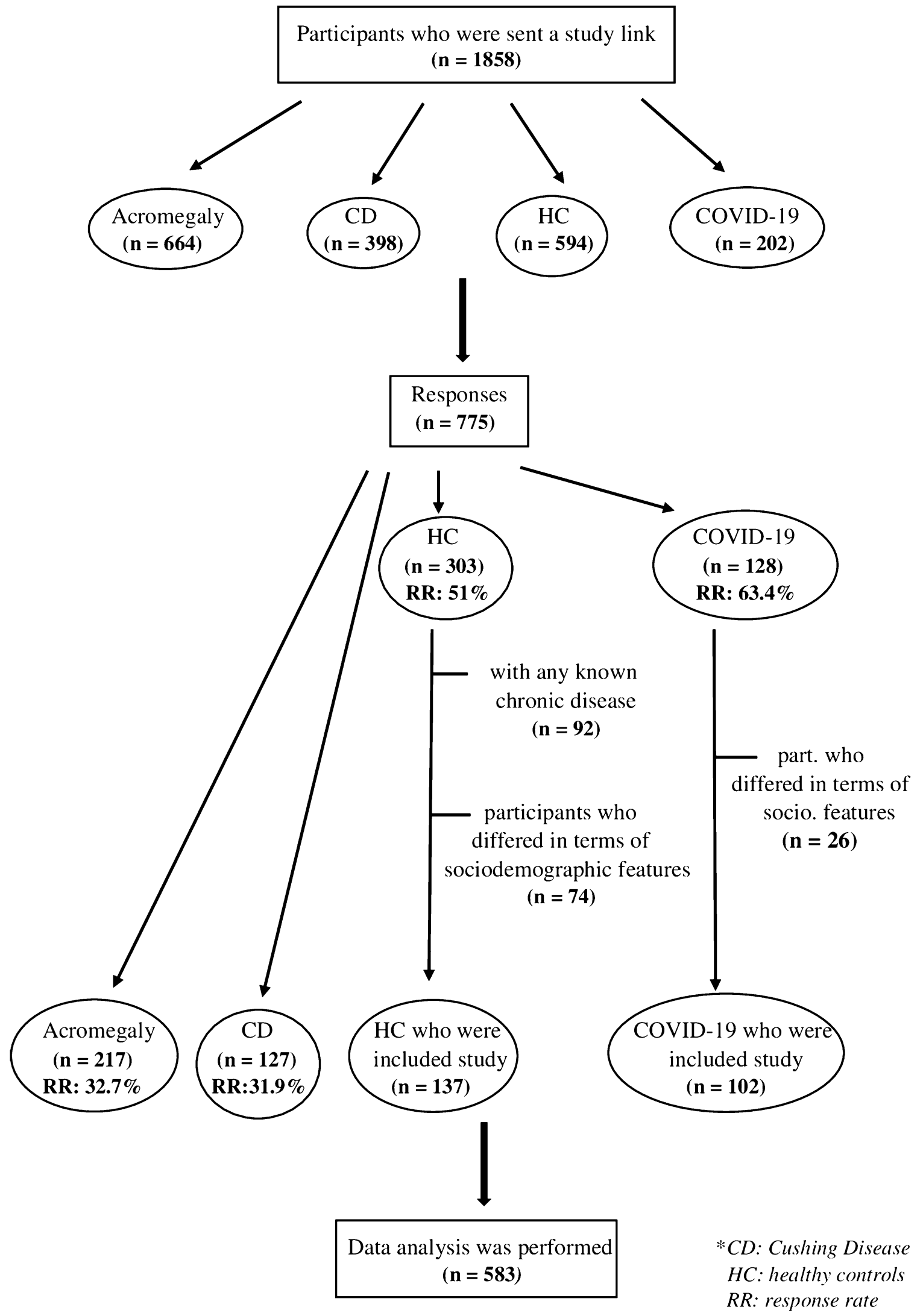

Fig. 1 The flowchart showing the patients and controls' recruitment process 
Fig. 2 The frequency of anxiety among groups according to the STAI-S and STAI-T



STAI-S: State-Trait Anxiety Inventory - State STAI-T: State-Trait Anxiety Inventory - Trait

Table 1 Sociodemographic characteristics of participants

\begin{tabular}{|c|c|c|c|c|c|}
\hline Characteristics & Acromegaly $(\mathrm{n}=217)$ & $\begin{array}{l}\mathrm{CD}(\mathrm{n}=127) \\
\text { Mean } \pm \mathrm{SD} \text { or } \\
\mathrm{n}(\%)\end{array}$ & COVID-19 $(\mathrm{n}=102)$ & Controls $(n=137)$ & $P$ \\
\hline Age & $47.5 \pm 11.1$ & $47.9 \pm 11.8$ & $50.3 \pm 11.5$ & $47.3 \pm 11.1$ & 0.162 \\
\hline \multicolumn{6}{|l|}{ Sex } \\
\hline Male & $97(44.7)$ & $27(21.3)$ & $53(52)$ & $62(45.3)$ & \multirow[t]{2}{*}{$<0.001 *$} \\
\hline Female & $120(55.3)$ & $100(78.7)$ & $49(48)$ & $75(54.7)$ & \\
\hline \multicolumn{6}{|l|}{ Marital status } \\
\hline Married & $169(77.9)$ & $89(70.1)$ & $87(85.3)$ & $113(82.5)$ & \multirow[t]{3}{*}{0.078} \\
\hline Single & $34(15.7)$ & $25(19.7)$ & $9(8.8)$ & $13(9.5)$ & \\
\hline Divorced & $14(6.5)$ & $13(10.2)$ & $6(5.9)$ & $11(8)$ & \\
\hline \multicolumn{6}{|c|}{ Years of education } \\
\hline$<12$ years & $163(75.1)$ & $93(73.2)$ & $77(75.5)$ & $87(63.5)$ & \multirow[t]{2}{*}{0.083} \\
\hline$>12$ years & $54(24.9)$ & $34(26.8)$ & $25(24.5)$ & $50(36.5)$ & \\
\hline \multicolumn{6}{|c|}{ Employment status } \\
\hline nemployed & $55(25.3)$ & $29(22.8)$ & $24(23.4)$ & $72(52.6)$ & \multirow[t]{2}{*}{$<0.001$} \\
\hline mployed & $162(74.7)$ & $98(77.2)$ & $78(76.6)$ & $65(47.4)$ & \\
\hline \multicolumn{6}{|c|}{ No. of people living together } \\
\hline Alone & $11(5.1)$ & $5(3.9)$ & $5(4.9)$ & $9(6.6)$ & \multirow[t]{4}{*}{0.124} \\
\hline $2-3$ & $110(50.7)$ & 77 (60.6) & $46(45.1)$ & 67 (48.9) & \\
\hline $4-5$ & $90(41.5)$ & $41(32.3)$ & $44(43.1)$ & $60(43.8)$ & \\
\hline$\geq 6$ & $6(2.8)$ & $4(3.1)$ & $7(6.9)$ & $1(0.7)$ & \\
\hline
\end{tabular}

CDCushing's disease

*When the patients with CD were excluded fromthe analysis, no significant difference was found between groups for sex $(P=0.451)$

individuals may have been more affected by the isolation and quarantine conditions during the COVID-19 outbreak.

Although acromegaly and CD may predispose individuals to psychosocial impairments, the rate of abnormal state anxiety in patients with acromegaly and CD was similar to the that of healthy controls. We also observed that acromegaly patients had higher levels of abnormal trait anxiety than healthy controls ( $70.5 \%$ of them). The rate of state 
Table 2 The comorbid diseases in patients with acromegaly and CD

\begin{tabular}{lccc}
\hline Variables, $\mathrm{n}(\%)$ & $\begin{array}{l}\text { Acromegaly } \\
(\mathrm{n}=217)\end{array}$ & $\mathrm{CD}(\mathrm{n}=127)$ & $P$ \\
\hline $\begin{array}{l}\text { Presence of any } \\
\text { comorbid disease }\end{array}$ & $107(49.3)$ & $71(55.9)$ & 0.285 \\
$\begin{array}{l}\text { Comorbidities } \\
\text { Diabetes mellitus }\end{array}$ & $58(26.7)$ & $33(26)$ & \\
Hypertension & $56(25.8)$ & $46(36.2)$ & 0.981 \\
OSAS & $30(13.8)$ & $7(5.5)$ & $\mathbf{0 . 0 4 1}$ \\
COPD & $15(6.9)$ & $15(11.8)$ & 0.120 \\
Heart failure & $9(4.1)$ & $8(6.3)$ & 0.374 \\
Cancer & $8(3.7)$ & $4(3.1)$ & 0.999 \\
Immunodeficiency & $2(0.9)$ & $4(3.1)$ & 0.273 \\
\hline
\end{tabular}

$C D$ Cushing's Disease, OSAS obstructive sleep apnea syndrome, $C O P D$ chronic obstructive pulmonary disease or trait anxiety in previous studies has been shown to vary between 10 and 13\% [27-29]. These rates were quite low compared to the rate found in our study. Our findings indicate that trait anxiety is slightly, but significantly, higher in acromegaly patients when compared to healthy controls during the COVID-19 pandemic. The anxiety, which has reached very high levels in patients with acromegaly during this period, may be associated with the use of mostly injectable drugs, which should be administered periodically by experienced healthcare professionals in the treatment of acromegaly, resulting in these patients' greater need for healthcare services. During the outbreak, the restrictions on access to hospitals for various reasons and the inability to receive medical treatment may have been stressful. In the literature, Giustina et al. revealed that more than one-quarter of

Table 3 Comparison of the groups in terms of IES-R and STAI scores

\begin{tabular}{|c|c|c|c|c|c|}
\hline Scales & Acromegaly $(\mathrm{n}=217)$ & $\begin{array}{l}\text { CD }(n=127) \\
\text { Mean } \pm S D\end{array}$ & COVID-19 $(\mathrm{n}=102)$ & Controls $(\mathrm{n}=137)$ & $P$ \\
\hline \multicolumn{6}{|l|}{$I E S-R$} \\
\hline Total & $23.2 \pm 16.3$ & $22 \pm 14.3$ & $34.8 \pm 12.4$ & $22 \pm 13.9$ & $<0.001 *$ \\
\hline Intrusion & $7.1 \pm 6.2$ & $6.7 \pm 5.9$ & $11.9 \pm 5.5$ & $7 \pm 5.1$ & $<0.001 *$ \\
\hline Avoidance & $10.3 \pm 6.3$ & $10.3 \pm 5.9$ & $14.3 \pm 4.9$ & $10.4 \pm 5.8$ & $<0.001 *$ \\
\hline \multicolumn{5}{|l|}{ STAI } & $<0.001 *$ \\
\hline STAI-S & $39.5 \pm 10.4$ & $38.8 \pm 10.8$ & $48.9 \pm 9.1$ & $38.9 \pm 11.5$ & $<0.001 *$ \\
\hline STAI-T & $44.4 \pm 9.2$ & $43.5 \pm 9.9$ & $46.1 \pm 7.3$ & $41.5 \pm 10.2$ & $\mathbf{0 . 0 0 2} \mathbf{2}^{\dagger}$ \\
\hline
\end{tabular}

CDCushing's disease, IES-R Impact of Event Scale-Revised, STAIState-Trait Anxiety Inventory

Post-hoc analysis results (adjusted p value in Bonferroni correction)

*COVID-19 vs. Acromegaly, $P<0.001$; COVID-19 vs. Cushing, $P<0.001$; COVID-19 vs. Controls, $P<0.001$

${ }^{\dagger}$ COVID-19 vs. Controls, $P=0.001$, Acromegaly vs. Controls, $P=0.049$

Fig. 3 The frequency of PTSD among groups according to the IES-R

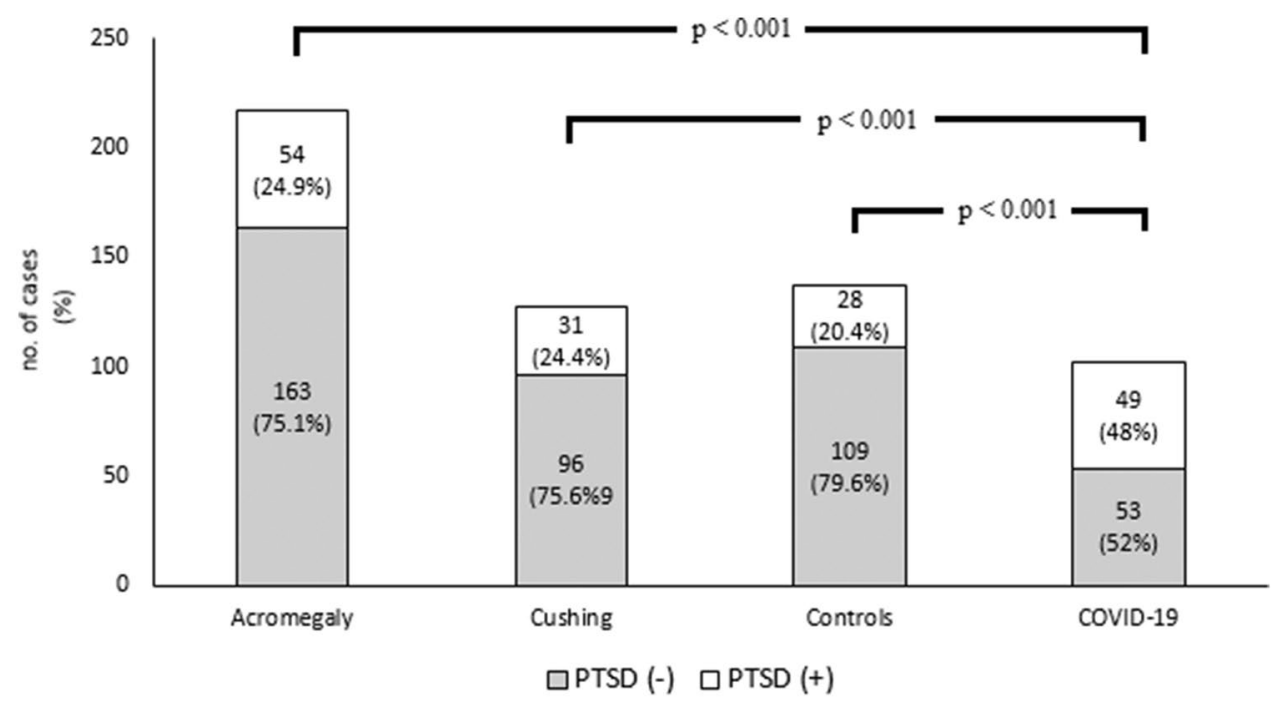

PTSD: posttra umatic stress disorders 
Table 4 Follow-up characteristics, risk perception for present diseases and problems with treatment in the COVID-19 outbreak

\begin{tabular}{|c|c|c|c|}
\hline Questions & $\begin{array}{l}\text { Acromegaly }(\mathrm{n}=217) \\
\mathrm{n}(\%) \text { or median }(\mathrm{IQR} 25-75)\end{array}$ & $\mathrm{CD}(\mathrm{n}=127)$ & $P$ \\
\hline \multicolumn{4}{|c|}{ Is the COVID-19 outbreak a very serious incident? } \\
\hline Yes & $160(73.7)$ & $101(79.5)$ & \multirow[t]{2}{*}{0.279} \\
\hline No & $57(26.3)$ & $26(20.5)$ & \\
\hline \multicolumn{4}{|c|}{ Consulting own doctor about COVID-19 } \\
\hline Yes & $39(18)$ & $22(17.3)$ & \multirow[t]{2}{*}{0.995} \\
\hline No & $178(82)$ & $105(82.7)$ & \\
\hline Last visit time (months ago) & $4(3-6)$ & $4(3-6)$ & 0.582 \\
\hline \multicolumn{4}{|c|}{ Missing outpatient clinic appointment } \\
\hline Yes & $100(46.1)$ & $63(49.6)$ & \multirow[t]{2}{*}{0.528} \\
\hline No & $117(53.9)$ & $64(50.4)$ & \\
\hline \multicolumn{4}{|c|}{ Missing this appointment is a problem? } \\
\hline Yes & $114(52.5)$ & $75(59.1)$ & \multirow[t]{2}{*}{0.289} \\
\hline No & $103(47.5)$ & $52(40.9)$ & \\
\hline \multicolumn{4}{|c|}{ Are having acromegaly/CD a risk for COVID-19? } \\
\hline Yes & $141(65)$ & $84(66.1)$ & \multirow[t]{2}{*}{0.919} \\
\hline No & $76(35)$ & $43(33.9)$ & \\
\hline \multicolumn{4}{|c|}{ Are medical treatment of acromegaly / CD a risk for COVID-19? } \\
\hline Yes & $18(8.3)$ & $13(10.2)$ & \multirow[t]{3}{*}{0.750} \\
\hline No & $167(77)$ & $91(71.7)$ & \\
\hline No any medication & $32(14.7)$ & $23(18.1)$ & \\
\hline \multicolumn{4}{|c|}{ Have acromegaly/CD medical treatment been discontinued? } \\
\hline Yes & $42(19.4)$ & $13(10.2)$ & \multirow[t]{3}{*}{0.012} \\
\hline No & $141(64.9)$ & $91(71.7)$ & \\
\hline No any medication & $34(15.7)$ & $23(18.1)$ & \\
\hline \multicolumn{4}{|l|}{ Problems in drug supply } \\
\hline Yes & $10(4.6)$ & $10(7.9)$ & \multirow[t]{3}{*}{0.286} \\
\hline No & $175(80.7)$ & $94(74)$ & \\
\hline No any medication & $32(14.7)$ & $23(18.1)$ & \\
\hline
\end{tabular}

patients reported difficulties accessing therapy, possibly supporting this anxiety [30]. This was the first study which had examined the real-world impacts of COVID-19 on patients with acromegaly; but the authors made no further mention of the psychosocial effects caused by this situation.

As for patients with $\mathrm{CD}$, anxiety rates varying between $12 \%$ [31] and $66 \%$ [32] were reported in patients with active CD, and a rate of $20 \%$ was reported for those in remission [33]. In addition, Dorn et al. revealed that anxiety disorders were present in $7.1 \%$ of the patients in the third month of their treatment, but that anxiety regressed in all patients after 6 to 12 months [34]. In light of these rates, although there were no significant differences found between patients with CD and healthy controls in terms of state or trait anxiety in our study, it can be concluded that anxiety levels in patients with CD increased during the outbreak period. Specifically, the levels were $58.4 \%$ for trait anxiety, $49.6 \%$ for state anxiety. We also found that the rates of abnormal state and trait anxiety were $84.3 \%$ and $85.3 \%$ respectively in PCR-confirmed COVID-19 patients; thus, significantly higher than all other groups. In the literature, a systematic review and meta-analysis shows that the prevalence of anxiety was the highest (56\%) among patients with COVID-19 in comparison to the general population [26]. On the contrary, Zhang et al. reported that there were no differences in anxiety levels between patients with COVID-19 and the general population [35].

A recent systematic review reported that the prevalence of PTSD in the general population during the COVID-19 outbreak ranged from 7 to $53.8 \%$ [36]. In our study, we found no significant differences in terms of PTSD (IES-R score $\geq 33$ ) between the acromegaly group (24.9\%), CD group $(24.4 \%)$ and healthy controls $(20.4 \%)$. This finding suggests that although having a chronic illness predisposes patients with acromegaly and CD to be strongly affected by COVID-19, they do not experience the pandemic as more traumatic than the general population. As a matter of fact, in a study by Poyraz et al. (2020), researchers evaluated 
the protracted psychiatric symptoms after COVID-19, and showed that the severity of PTSD symptoms did not change to the presence of a chronic medical disease [37]. Patients with PCR-confirmed COVID-19 had significantly higher rates $(48 \%)$ of PTSD than the other three groups. Among the 714 hospitalized but stable COVID-19 patients, the rate of PTSD was found to be $96.2 \%$ [38]. We may say that contracting COVID-19 is a very traumatic experience due to the fact that patients feel they will face many additional catastrophic situations.

In our study, there may be other reasons for the lack of differences in terms of state-anxiety and PTSD levels between patients with acromegaly, patients with $\mathrm{CD}$ and healthy controls. Most of the patients with acromegaly or $\mathrm{CD}$ had regular follow-up visits and the median last visit time was 4 months prior to the survey. Approximately twothirds of the patients in both groups considered acromegaly and CD a risk for COVID-19 and took the necessary precautions. $90 \%$ of patients with acromegaly or CD did not consider treatment of the present diseases as a risk and the discontinuation rate of present medical treatment did not exceed $20 \%$. As a result of all this, we can say that the risk perception related to COVID-19 has decreased due to regular follow-up visits and medical treatment and that the psychopathological effects of the pandemic are similar between the patients and the rest of society. However, in both the acromegaly and CD groups, it can be extrapolated that the perceived threat disappeared as a result of lessened contact with COVID-19. The fact that no patients or their family members contracted COVID-19 at a higher rate or severity than the general population may have resulted in less anxiety and PTSD as well.

In the present study, several limitations should be considered. First, in our web-based study, the participants were not obligated to fill in descriptive information such as name, surname or patient ID numbers for the ethical reasons. Therefore, we could not reach all of the medical record data regarding the clinical features, biochemical findings and remission status of patients with acromegaly and CD. This could have offered the opportunity to evaluate the psychological conditions of patients depending on their disease activity. Second, we excluded in blinded manner respondents who differed from patients in terms of socioeconomic features. This may have biased our results. On the other hand, this approach gave us the opportunity create more homogenous and similar groups and decrease confounding factors. Third, due to the female preponderance among patients with CD [39], we formed control groups similar to only patients with acromegaly for sex. Finally, as a feature of survey studies, the presence of chronic disease may not reflect the actual situation as it was collected based on selfreport of the participants.
In conclusion, we demonstrated that patients with acromegaly and CD experienced similar impacts when compared to healthy controls during the COVID-19 outbreak in terms of anxiety and PTSD. The abnormal trait anxiety in patients with acromegaly was higher compared to healthy controls. In addition, having a confirmed diagnosis of COVID-19 linked to the most heightened state of both anxiety and PTSD development. In light of these findings, clinicians should consider the psychosocial effects of the pandemic and focus on the regular follow-up and medical treatments of these patients during the outbreak.

Author contributions All authors made substantial contributions to the conception and design, and/or acquisition of data, and/or analysis and interpretation of data; participated in drafting the article or revising it critically for important intellectual content; and gave final approval of the version to be submitted.

Funding This research did not receive any specific grants from any funding agencies in the public, commercial, or non-profit sector.

Data availability The data that support the findings of this study are available from the corresponding author upon reasonable request.

\section{Declarations}

Conflict of interest The authors declare no conflicts of interest that could be perceived as prejudicing the impartiality of the research reported.

Ethical approval The study was approved by the local ethics committee of the Istanbul University-Cerrahpasa, Cerrahpasa Medical Faculty (Decision No: 56321 dated 22 April, 2020). And all procedures performed in studies involving human participants were in accordance with the ethical standards of the institutional and/or national research committee and with the 1964 Helsinki declaration and its later amendments or comparable ethical standards.

Informed consent Informed consent was obtained from all individual participants included in the study.

\section{References}

1. Johns Hopkins University (2021) COVID-19 Dashboard by the Center for Systems Science and Engineering (CSSE) at Johns Hopkins University (JHU). https://gisanddata.maps.arcgis.com/ apps/opsdashboard/index.html\#/bda7594740fd40299423467b4 8e9ecf6. Accessed 23 Jan 2021

2. Baj J, Karakuła-Juchnowicz H, Teresiński G et al (2020) COVID19: specific and non-specific clinical manifestations and symptoms: the current state of knowledge. J Clin Med 9:1753. https:// doi.org/10.3390/jcm 9061753

3. Roberts CM, Levi M, McKee M et al (2020) COVID-19: a complex multisystem disorder. Br J Anaesth 125:238-242

4. Wang W, Xu Y, Gao R et al (2020) Detection of SARS-CoV-2 in different types of clinical specimens. JAMA 323:1843-1844 
5. Yuki K, Fujiogi M, Koutsogiannaki S (2020) COVID-19 pathophysiology: a review. Clin. Immunol. 215:108427

6. Republic of Turkey Ministry of Health (2020) Republic of Turkey Ministry of Health Corona Table. https://covid19.saglik.gov.tr/. Accessed 12 Jan 2020

7. Wang C, Pan R, Wan X et al (2020) Immediate psychological responses and associated factors during the initial stage of the 2019 coronavirus disease (COVID-19) epidemic among the general population in China. Int J Environ Res Public Health. https://doi. org/10.3390/ijerph17051729

8. Özdin S, Bayrak Özdin Ş (2020) Levels and predictors of anxiety, depression and health anxiety during COVID-19 pandemic in Turkish society: the importance of gender. Int J Soc Psychiatry 66:504511. https://doi.org/10.1177/0020764020927051

9. Ozamiz-Etxebarria N, Dosil-Santamaria M, Picaza-Gorrochategui M, Idoiaga-Mondragon N (2020) Stress, anxiety, and depression levels in the initial stage of the COVID-19 outbreak in a population sample in the northern Spain. Cad Saude Pub. https://doi. org/10.1590/0102-311X00054020

10. Oner N, LeCompte A (1985) Durumluk-Sürekli Kayg1. El Kitab1 (State-Trait Anxiety Inventory Handbook), 2.Baskı. Boğaziçi Üniversitesi Yayınları, Istanbul

11. Weiss DS (1997) The impact of event scale-revised. In: Wilson JP, Keane TM (eds) Assessing Psychological Trauma and PTSD. Guilford Press, New York, pp 399-411

12. Morina N, Ehring T, Priebe S (2013) Diagnostic utility of the impact of event scale-revised in two samples of survivors of war. PLoS ONE. https://doi.org/10.1371/journal.pone.0083916

13. Reynolds DL, Garay JR, Deamond SL et al (2008) Understanding, compliance and psychological impact of the SARS quarantine experience. Epidemiol Infect 136:997-1007. https://doi.org/10.1017/ S0950268807009156

14. Longo P, Bertorello A, Panero M et al (2019) Traumatic events and post-traumatic symptoms in anorexia nervosa. Eur J Psychotraumatol. https://doi.org/10.1080/20008198.2019.16829302019.1682930

15. Seyahi E, Poyraz BC, Sut N et al (2020) The psychological state and changes in the routine of the patients with rheumatic diseases during the coronavirus disease (COVID-19) outbreak in Turkey: a web-based cross-sectional survey. Rheumatol Int 40:1229-1238. https://doi.org/10.1007/s00296-020-04626-0

16. Wang C, Pan R, Wan X et al (2020) A longitudinal study on the mental health of general population during the COVID-19 epidemic in China. Brain Behav Immun 87:40-48. https://doi.org/10.1016/j. bbi.2020.04.028

17. Alkhamees AA, Alrashed SA, Alzunaydi AA et al (2020) The psychological impact of COVID-19 pandemic on the general population of Saudi Arabia. Compr Psychiatry. https://doi.org/10.1016/j.compp sych.2020.152192

18. Bohlken J, Schömig F, Lemke MR et al (2020) COVID-19 pandemic: stress experience of healthcare workers: a short current review. Psychiatr Prax 47:190-197

19. Chew NWS, Lee GKH, Tan BYQ et al (2020) A multinational, multicentre study on the psychological outcomes and associated physical symptoms amongst healthcare workers during COVID-19 outbreak. Brain Behav Immun 88:559-565. https://doi.org/10.1016/j. bbi.2020.04.049

20. Creamer M, Bell R, Failla S (2003) Psychometric properties of the Impact of Event Scale-revised. Behav Res Ther 41:1489-1496. https ://doi.org/10.1016/j.brat.2003.07.010

21. Çorapçioğlu A, Yargiç İ, Geyran P, Kocabaşoğlu N (2006) "Olaylarin etkisi ölçeği” (IES-R) Türkçe versiyonunun geçerlilik ve güvenilirliği. = Validity and reliability of Turkish Version of "Impact of Event Scale-Revised" (IES-R). Yeni Symp Psikiyatr nöroloji ve davraniş Bilim Derg
22. Zhang L, Zhu F, Xie L et al (2020) Clinical characteristics of COVID-19-infected cancer patients: a retrospective case study in three hospitals within Wuhan, China. Ann Oncol 31:894-901. https ://doi.org/10.1016/j.annonc.2020.03.296

23. Mazza C, Ricci E, Biondi S et al (2020) A nationwide survey of psychological distress among Italian people during the covid-19 pandemic: Immediate psychological responses and associated factors. Int J Environ Res Public Health: https://doi.org/10.3390/ijerp h17093165

24. Shi L, Lu ZA, Que JY et al (2020) Prevalence of and risk factors associated with mental health symptoms among the general population in China during the coronavirus disease 2019 pandemic. JAMA Netw Open. https://doi.org/10.1001/jamanetworkopen.2020.14053 2020.14053

25. Salari N, Hosseinian-Far A, Jalali R et al (2020) Prevalence of stress, anxiety, depression among the general population during the COVID-19 pandemic: a systematic review and meta-analysis. Glob. Health 16:57

26. Luo M, Guo L, Yu M, Wang H (2020) The psychological and mental impact of coronavirus disease 2019 (COVID-19) on medical staff and general public; a systematic review and meta-analysis. Psychiatry Res. 291:113190

27. Sievers $\mathrm{C}$, Ising M, Pfister $\mathrm{H}$ et al (2009) Personality in patients with pituitary adenomas is characterized by increased anxiety-related traits: Comparison of 70 acromegalic patients with patients with non-functioning pituitary adenomas and age- and gender-matched controls. Eur J Endocrinol 160:367-373. https://doi.org/10.1530/ EJE-08-0896

28. Sievers C, Dimopoulou C, Pfister H et al (2009) Prevalence of mental disorders in acromegaly: A cross-sectional study in 81 acromegalic patients. Clin Endocrinol (Oxf) 71:691-701. https://doi.org/10 $.1111 / \mathrm{j} .1365-2265.2009 .03555 . x$

29. Crespo I, Santos A, Valassi E et al (2015) Impaired decision making and delayed memory are related with anxiety and depressive symptoms in acromegaly. Endocrine 50:756-763. https://doi.org/10.1007/ s12020-015-0634-6

30. Giustina A, Mario CB, Marks M et al (2020) AcroCOVID: an international survey on care for acromegaly during the COVID-19 era. Endocr Abstr. https://doi.org/10.1530/endoabs.70.aep1074

31. Kelly WF (1996) Psychiatric aspects of Cushing's syndrome. QJM Mon J Assoc Phys 89:543-551. https://doi.org/10.1093/qjmed /89.7.543

32. Starkman MN, Schteingart DE, Schork MA (1981) Depressed mood and other psychiatric manifestations of Cushing's syndrome: relationship to hormone levels. Psychosom Med 43:3-18. https://doi. org/10.1097/00006842-198102000-00002

33. Tiemensma J, Biermasz N, Middelkoop H et al (2010) Increased prevalence of psychopathology and maladaptive personality traits after long-term cure of Cushing's disease 31:608-609

34. Dorn LD, Burgess ES, Friedman TC et al (1997) The longitudinal course of psychopathology in Cushing's syndrome after correction of hypercortisolism. J Clin Endocrinol Metab 82:912-919. https:// doi.org/10.1210/jc.82.3.912

35. Zhang J, Lu H, Zeng $\mathrm{H}$ et al (2020) The differential psychological distress of populations affected by the COVID-19 pandemic. Brain Behav Immun 87:49-50

36. Xiong J, Lipsitz O, Nasri F et al (2020) Impact of COVID-19 pandemic on mental health in the general population: a systematic review. J Affect Disord 277:55-64

37. Poyraz B, Poyraz CA, Olgun Y et al (2020) Psychiatric morbidity and protracted symptoms after COVID-19. Psychiatry Res 295:113604. https://doi.org/10.1016/j.psychres.2020.113604

38. Bo HX, Li W, Yang Y et al (2020) Posttraumatic stress symptoms and attitude toward crisis mental health services among clinically 
stable patients with COVID-19\&nbsp;in China. Psychol Med. https ://doi.org/10.1017/S0033291720000999

39. Boscaro M, Barzon L, Fallo F, Sonino N (2001) Cushing's syndrome. Lancet 357:783-791. https://doi.org/10.1016/S0140 $-6736(00) 04172-6$
Publisher's note Springer Nature remains neutral with regard to jurisdictional claims in published maps and institutional affiliations. 